\title{
Influence of Social Broadcasting Networks on News Consumption
}

\author{
Shailesh Palekar \\ Queensland University of Technology \\ s.palekar@qut.edu.au
}

\section{Darshana Sedera}

Monash University

\section{Abstract}

This study assesses potentially sensitive effects of a specific sub-class of digital social technologies called social broadcasting networks (SBNs). Adopting the explanatory mechanisms of social network structures (Borgatti et al. 2009; Kane et al. 2014), and adapting Herzberg et al's (1959) Two Factor theory, it investigates how the structure of an SBN (conceptualized as its relative advantage), and the influence of users in an SBN empower two key news consumption behaviours viz. switching and continuance. Employing the survey (qualitative) method, the study empirically validates the news consumption behaviours of 325 respondents. Findings reveal a more nuanced role of SBNs indicating the potential benefits to news firms because of individuals switching to traditional news mediums while in the same breadth highlights the potential of SBNs as competitive news providers. It shows intermittent use of SBNs and signifies that when online and offline news mediums co-exist, influence of users in the incumbent (online) medium plays a powerful role in inducing or refraining the use of a substitute offline medium. This study develops new ontological and theoretical understanding of the entanglement of technology and social activity that is dynamic, at times ephemeral and pervasively ubiquitous.

Keywords Social Media; Two-Factor Theory; News Media

\section{Introduction}

Digital social technologies such as Facebook and Twitter have also been defined as social broadcasting networks (SBNs) (Shi et al. 2014). On a more holistic and higher-order level they simultaneously provide a broadcasting service as well as enable social networking (Shi et al. 2014). SBNs broadcast information similar to traditional subscribed relationships through followers-following on Twitter, friends on Facebook or groups in LinkedIn (Shi et al. 2014). SBNs focus on converging micro-blogging, user-generated-content and social networking (Kang and Lee 2017). For example, onlooker Janis Krums tweeted the dramatic crash landing of U.S. Airways Flight 1549 on the frigid waters of the Hudson River as it unfolded in real time (Krums 2009). His tweet was quickly consumed and re-tweeted by hundreds of other Twitter users even before the news media caught wind of the dramatic incident. Thus, SBNs blur the contemporary precincts of information-based businesses such as traditional news mediums (e.g. television, newspapers) due to its (i) decentralized structure converged by a diverse spectrum of information ( $\mathrm{Wu}$ et al. 2011) (ii) open and loose network of people that are unilaterally established with ease across geographical zones (Shi et al. 2014), and (iii) freedom over information creation, dissemination and consumption (Kwak et al. 2010; Palekar et al. 2015). News consumer are no longer tied to any specific news mediums (e.g. television, online news website). Rather, they traverse through diverse online news sources and different SBNs 
(Pew Research Center 2013; Williamson et al. 2012) expecting and demanding news in fast, convenient and smart ways consummate with a deep social and human touch. By amalgamating computing, communication and connectivity technologies (Bharadwaj, El Sawy, Pavlou, \& Venkatraman, 2013; Oestreicher-Singer \& Zalmanson, 2013), SBNs have deeply impacted news consumption by creating novel ways for communicating and creating newsworthy information (Bruns, Highfield, \& Ann Lind, 2012; Pentina \& Tarafdar, 2014).

This study investigates how SBNs and user influence in such networks affect the news consumption of people. Media and user influence effects are important for understanding the nomological net of information systems (viz. SBNs in this paper) and explicating the opportunities or threats they pose. Online viral effects of news consumption, diffusion and dissemination exhibit actions similar to real-life contagion behaviours such as spontaneity, unpredictability, irrationality or disorderliness (Coleman 1990; Sedera et al. 2016b). Anecdotal commentary suggests that user influence and affinity spawned by mass individuals in SBNs result in the continuance of news consumption in the networks and aid their evolving roles as powerful platforms for broadcasting, sourcing and consuming news. Thus, it is germane to investigate SBNs for developing new ontological and theoretical understanding of the entanglement of technology and social activity that is dynamic, at times ephemeral and pervasively ubiquitous. Specifically, this paper investigates a key research question: What factors influence news consumption on social broadcasting networks?

\subsection{News Consumption Behaviours on Social Broadcasting Networks}

SBNs render users the choice and freedom to switch news consumption to substitutable news mediums based on the utility and gratifications afforded by the medium (Kang and Lee 2017; Yuan 2011). For example, individuals reading news tweeted by a news firm can instantly switch to the news firms' website by clicking on the web links provided with the news tweet. Likewise, individuals on SBNs can also switch their news consumption to other Internet-based (e.g. news website) or offline news mediums (e.g. television) based on social and psychological needs for selecting a specific medium or news content area (Diddi \& LaRose, 2006) for more information and news verification. In such transformative trends, consumers dictate key elements serving their consumption choices, which include demand and control over how, when and where news should be consumed and ownership of self-generated content (Setia et al. 2013). Thus, the relative advantage of SBNs in facilitating (i) open access and connectedness to information and its providers (ii) ownership of information (iii) immediacy of news, and (iv) control over what, when and how news is consumed, challenges the "dialectic tensions between the public-interest role of the news media" (Hirst 2011, p. 15). In SBNs, users exerting influence on other users in SBNs is a critical driver of mass consumption and the networks' successful functioning (Shi et al. 2014). Such mass information exchange creates a "web of cooperative relationships that breeds norms, trust, common purpose, and coordination - that is social capital" (Kane et al. 2014, p. 277). People of repute (e.g. entertainers, business and political leaders) frequently use SBNs to connect with all stakeholders, garner, reclaim and retain public support and trust, and influence future social, business and political ideas, agenda's and trends. This provides users in SBNs the opportunity to connect with such personalities, which otherwise is difficult in the real world. Consuming such content provides individuals "information, influence, or social support" (Kane et al. 2014, p. 277). News practitioners (e.g. reporters) also utilize SBNs, to instantly disseminate news, and influence people to engage with the news companies they represent. Past studies on post-adoption use of IT treat continuous use or the decision for continuing the use of an innovation, technology 
or product as similar (Sedera et al. 2016a; Ye et al. 2008). Such treatment relies on the assumption that specific technologies (e.g. Web 2.0 applications) represent the innovation (i.e. SBNs). This is a reasonable assumption based on the setting of many digital social technologies (e.g. SBNs) that have multiple products, which are similar in functionality and substitutability (e.g. Yammer and LinkedIn). However, in the context of this study, users switching news consumption from SBNs (e.g. Twitter) to a substitute that serves similar needs, such as a television news channel or a news website constitutes partial or intermittent replacement of news mediums fulfilling similar needs (Bhattacherjee et al. 2012). In such scenarios, users opt for both, SBNs and offline news mediums on a concurrent basis.

Based on the above, this study develops and tests a model depicting individuals (i) switching their news consumption from SBNs to traditional news firms, and (ii) continuing their news consumption on SBNs. The research model serves as a strong start-up for understanding IT switching as well as continuance behaviours in the same breadth by incorporating the supply side effects (e.g. availability of related products) as well as the demand side effects (e.g. user needs and preferences). Its main goal is to empirically evidence changes in news consumption behaviours based on the influence of SBNs (i.e. its advantages as a medium) and influence in SBNs (i.e. users influencing other users in SBNs). The issues discussed provide the premise and the motivation for exploring how SBNs disrupt the ways in which people understand and enhance their news creation and consumption experiences. In addition, the defiant roles of SBNs in disrupting traditional ways of consuming news and threating the identity of traditional news firms (Kietzmann et al. 2011) has been largely under-researched or overlooked in past IS studies, which this paper explores. Thus, this study responds to calls for researching the strengths and drawbacks of digital social technologies and theoretically explain their mechanisms and dynamics. This paper is further structured as follows: It begins with theoretical foundations followed by the section on the process model development. Next, it details hypothesis development and methodology followed by data analysis and findings. It concludes with contributions to research and implications for practice and then lists the study limitations.

\section{Theoretical Foundations}

For analysing digital social networks, Borgatti \& Foster (2003) offer a parsimonious framework that describes four canonical types of explanatory mechanisms consisting of explanatory goals, which are built around the structure of a network and the content in the network. These explanatory goals capture the social homogeneity or disparities in network performance and performance variations (Kane et al. 2014, p. 277). SBNs offer users media choices that are motivated by a networks' structure, content and users. Thus, this study adopts the explanatory mechanisms centred around the (i) structure of an SBN, which relates to its relative advantage for understanding the social homogeneity in consuming news, and (ii) influence of users in an SBN for understanding how user performance impacts news consumption in an SBN. This provides the foundation in building up the factors that affect news switching and continuance behaviours.

\subsection{Relative Advantage of Social Broadcasting Networks}

Relative advantage is conceptualized as a multi-dimensional construct (Choudhury and Karahanna 2008) based on the diffusion of innovation theory (Refer to Rogers 1995). Through the diffusion of innovation theory, relative advantage captures the gains and benefits of an 
innovation on multiple dimensions such as increase in comfort levels, saving on costs, time and efforts (Lokuge and Sedera 2014a; Rogers 1995), or trust and convenience (Choudhury and Karahanna 2008). Underlining Rogers (1995 diffusion of innovation theory and cited by Choudhury and Karahanna (2008, p. 180), relative advantage is defined as "the degree to which an innovation is perceived as being better than using the practice it supersedes." A study by Bhattacherjee et al. (2012) applying the relative advantage construct for understanding user's IT switching behaviour find that performance expectancy based on the unified theory of acceptance and use of technology (Venkatesh et al. 2012) is a "stable and consistent predictor of IT acceptance over time" (Bhattacherjee et al. 2012, p. 328). A study on user's IT switching behaviour by Ye et al. (2008) find that the relative advantage of a competing IT product or service is positively related to users switching usage of existing IT products to a substitute that satisfies similar needs. This study adopts the relative advantage constructs as the best predictor of media choice in a post-adoption IT usage setting. It associates the relative advantage of SBNs through its value propositions relating to the (i) immediacy of news (Kietzmann et al. 2011) (ii) timeliness of news (Pew Research Center 2013), and (iii) connectedness to global issues (Kietzmann et al. 2011).

\subsection{User Influence in Social Broadcasting Networks}

The mass dissemination and sharing of user-generated-content in real time (Kaplan and Haenlein 2012) provides a unique perspective of how users influence news consumption (Lee and Ma 2012) in SBNs. The theoretical lens of social impact (Latané 1981) evidences that individuals are influenced by the actions and expectations of other individuals. Such influence is based on the (1) strength of the social force - i.e. the importance of the influencer group to the individual (2) immediacy of the social force - i.e. the temporal or physical proximity of the influencer group to the individual at the time of influence, and (3) number of people comprising the influencer group (Latané 1981). Thus, this study defines user influence as the extent to which a user can cause an effect in an indirect or intangible way on other users to follow or act in a certain way.

\subsection{News Consumption Behaviours in Social Broadcasting Networks}

This study defines news consumption on SBNs as diverse activities undertaken by users of SBNs, which involves interacting with information. These activities range from (1) posting text, photos, videos, random ideas, reading (surfing, browsing), sharing content, and commenting (Alarifi and Sedera 2014; Xu et al. 2012) (2) tweeting (Kwak et al. 2010), (3) listing (consuming information by grouping other users) (4) following (other users) (Alarifi et al. 2015) and, (4) subscribing (Velichety and Ram 2013).

Switching: Switching involves either partially or fully replacing the use of an IT service, application or product with a substitute that potentially fulfils similar needs and objectives (Bhattacherjee et al. 2012; Ye et al. 2008). However, IT switching may not involve the user in completely abandoning the existing use of IT artefacts or services but concurrently using diverse yet competing IT products and services based on users' interests, necessities and needs (Ye et al. 2008). This study contextualizes switching as concurrent or alternating news consumption between SBNs and traditional news mediums (e.g. television). It defines switching as, users' reducing their news consumption on an incumbent news medium (viz. SBNs) while replacing it in part with an alternate news medium (viz. traditional news mediums) that fulfils identical needs. The concept of switching is underlined by the continuance-discontinuance paradigm (Bhattacherjee et al. 2012; Ye et al. 2008) in a post-adoption IT/IS use settings where 
adoption, use and familiarity with the new product or service has already taken place (Ye et al. 2008). This study posits that the influence of users in SBNs and the relative advantage of SBNs significantly impact user switching behaviour in consuming news on SBNs.

Continuance: Continuance of news consumption in SBNs (and other online mediums) is important and critical for the sustenance and survival of news companies and is dependent on existing customers and their recurring demand for goods and services (Bhattacherjee 2001). Continuance research in IS has been examined through different ways such as for example, implementation (Zmud 1982), and infusion and routinization (Cooper and Zmud 1990). Past studies draw on several models for explaining IT continuance. For example, Hsu et al. (2014) investigate users' extrinsic and intrinsic motivations for explaining the continuing use of social networking sites by integrating the technology acceptance model (Davis 1989) and the theory of planned behaviour (Venkatesh and Davis 2000). The theoretical constructs that explain intentions for technology acceptance (e.g. ease of use) are generally applied as natural extensions for describing IT continuance. Lin and Huang (2014) propose a conceptual model explaining factors based on the pushing, pulling and mooring effects. This study defines continuance as persistently consuming news on an incumbent news medium while not replacing it with an alternate news medium that fulfils similar needs. It extends the existing IT/IS continuance literature by studying the influence of an SBN and influence in an SBN on a user rather than user intentions, motivations or cognitions.

\subsection{Two-Factor Theory}

This study uses the two-factor theory as the overarching theoretical lens to explain two factors (viz. influence of SBNs - relative advantage, and influence in SBNs - user influence) that influence two opposing news behaviours of switching and continuance. Psychologist Frederick Herzberg theorized that satisfaction and dissatisfaction in the workplace are caused by a set of factors that are independent of each other (Herzberg et al. 1959) (Also refer Park and Ryoo 2013). The two set of factors correspond to two opposing behaviours, intentions, or state of mind. Each set of factors and its corresponding impact variable are like two sides of a coin. They do not impact each other but are neutral to each other (Hulin and Smith 1967). Based on Herzberg et al. (1959) study, as dependent constructs in the theory are unrelated, one does not increase as the other diminishes. Although the two-factor theory has been applied in diverse research streams (e.g. consumer research (Maddox 1981), human behaviour (Park and Ryoo 2013)), its adoption and adaption in IS research is fairly recent (Cenfetelli and Schwarz 2010; Lee et al. 2009; Liu et al. 2011; Park and Ryoo 2013). In IS studies, the theory is referred to factors that encourage or provoke and prevent users towards adopting a product or service (Cenfetelli and Schwarz 2010). However, most studies explain only one side of user behaviour by looking at the set of factors that prevent and enable it, but in different contexts.

\section{The Process Model}

Based on the above, this study proposes a process model (Refer to Figure 1) for illustrating the progression of news consumption and its lead up to the comparative duration of news consumption in SBNs. The process model depicts how an individual on an SBN forms two distinct behaviours in response to prevailing conditions in the network.

First, an individual in an SBN wishing to acquire more information and be connected to global issues seeks information as events unfold in real time. This triggers a rational decision-making process influenced by the relative advantage of SBNs. The 
individual either (i) continues consuming news on the network [based on postadoption studies on IT continuance (Ortiz de Guinea and Markus 2009)], or (ii) switches to traditional news mediums for more information or verification [based on the post-adoption studies on IT switching behaviour (Ye et al. 2008)].

Second, when an individual in an SBN sees news, posts or updates by friends, other users or news firms, the same rational decision-making process (as stated above) occurs leading the individual to either continue in the network or switch to a traditional news medium.

The above observations help in conceptualising the antecedents of the two opposing news behaviours (i.e. switching news consumption to traditional news mediums and continuing news consumption in SBNs). The influence of SBNs through its value propositions of news immediacy (i.e. individuals can consume news whenever desired), timeliness (i.e. news is available as it happens) and connectedness (i.e. individuals can create, comment or share news thereby connecting to content and other individuals) reflect the relative advantage of the network. Influence in SBNs is observed through users influencing other users in SBNs. Both behaviours have an overall impact on the comparative duration of news consumed in SBNs thus highlighting the significance of SBNs. The process model describes users on SBN influenced by the network as well as users in the network. This is depicted through two situations which users generally face on SBNs. These further translate into two outcomes, which are depicted through the news behaviours of switching and continuance. The two-factor theory is illustrated for understanding the relationships between the influence of SBNs and the influence in SBNs on the news behaviours of switching and continuance. Although, as per the theory, each factor has a relationship with only one side of an individual's state of mind, behaviour or intent, this study extends the theory by testing the relationship of both factors on both news behaviours of switching and continuance. These news behaviours reflect the comparative duration of news consumption in SBNs, which is included in the research model to test the theory in its nomological net.

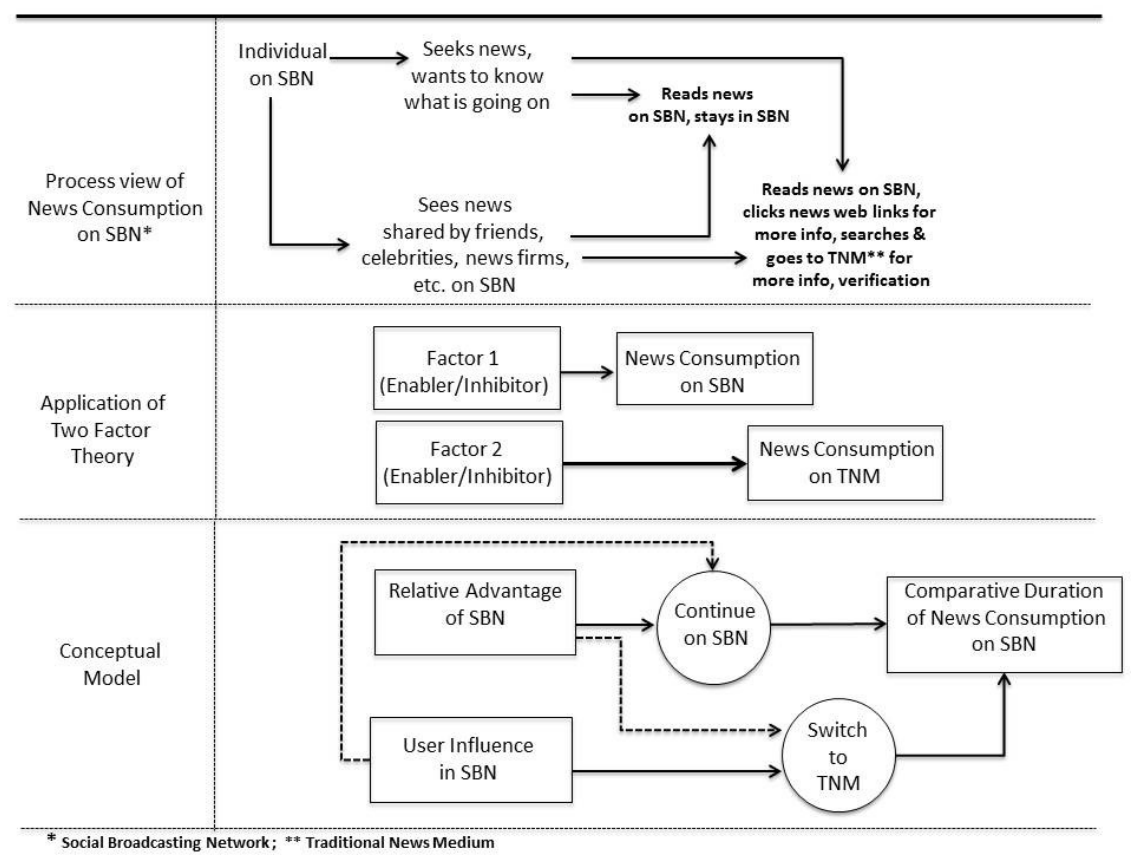

Figure 1. Process Model of News Consumption on SBN 


\section{Hypothesis Development}

The ubiquitous nature of SBNs as public networks has necessitated this study to look at two key factors constituting an important and integral part of the network itself. This differs from previous studies that have utilized the two-factor theory for understanding typically only one behaviour such as switching in a bounded organisational environment. The two factors used in this study are:

1. Influence of SBNs: This relates to the relative advantage of SBNs, which in turn is based on the premise of the "feature-centric view of technology" (Jasperson et al. 2005, p. 527). Technology features are interpreted as well as adapted by IT users in order to "constitute a technology-in-use" (Jasperson et al. 2005, p. 529) in post-adoption IT contexts. [Also refer to DeSanctis and Poole (1994)]. Technology features and their value propositions are important, as they influence and determine work outcomes at any given points in time (DeSanctis and Poole 1994). In addition, technology attributes reflect the "core of the technology, collectively representing its identity" (Jasperson et al. 2005, p. 529). Thus, the relative advantage of SBNs over their traditional counterparts (i.e. traditional news mediums) delineates the value propositions offered by the network for influencing user behaviour.

2. Influence in SBNs: This factor is associated with user influence in SBNs. These networks comprise a public environment wherein news consumption is a voluntary activity. The networks constitute large online communities (e.g. groups, associations, friends) with common shared interests (Ren et al. 2012). Individuals in such networks create and share information with others in the network (Wasko and Faraj 2005) making the networks important digital spaces for professional, political and social discussions (Ren et al. 2012). Social psychological theory informs the formation of individual attachments in a group, which happen through (1) the group's identity whereby people are attracted and feel connected to each other based on their goals, activities and character of the group (Hogg and Turner 1985), and (2) interpersonal bonds, friendships and other social relations developed with other members of the group. User influence on SBNs follow the notion of bond-based attachment arising from the exchange of personal and social information (Ren et al. 2012). This shifts the attention from the identity of the network to its individual members.

\subsection{Relative Advantage}

Relative advantage is conceptualized as a multi-dimensional construct (Choudhury and Karahanna 2008) based on the diffusion of innovation theory (Refer to Rogers 1995). Taking a feature-centric view (Jasperson et al. 2005) of SBNs, this study observes three prime features that reflect the core advantage of SBNs and represents the gratifications they offer:

1. Immediacy of newsworthy information (Kietzmann et al. 2011): Individuals use SBNs as they (i.e. the networks) transcend temporal and geographical boundaries and provide immediate access to information ( $\mathrm{Xu}$ et al. 2012). These networks facilitate conversations within network users creating instant pathways for information dissemination (Kietzmann et al. 2011)

2. Timeliness of newsworthy information ( $\mathrm{Li}$ and $\mathrm{Ng}$ 2013): Recent studies on user behaviours on Twitter find that instant news reporting (e.g. breaking news, headline news) and commenting on current events are some of the main intentions for using 
Twitter (Kwak et al. 2010; Li and Ng 2013). Kwak et al. (2010 exploring the topological attributes of Twitter finds that more than $80 \%$ of trending topics are headline news occurring in real life. Likewise, creating real-time status updates on SBNs (e.g. Facebook) creates an ambient cognizance of issues and events (Kaplan and Haenlein 2012), and

3. Connectedness (Kietzmann et al. 2011). These networks have become platforms of influence by engendering radically new ways of interacting and connecting with both, users and information (Hanna et al. 2011).

A study by Huang (2009) finds that young adults see digital news mediums (e.g. online websites, Facebook) as important platforms for connecting and staying informed on the world around them. Social connectedness that enhances information sharing in SBNs is limited in traditional news mediums in the absence of active and effectual diffusion channels (Lee and Ma 2012). SBNs also support four different types of ties or simple connections between users (Kane et al. 2014) such as (1) proximities representing shared physical or social spaces for ties to form (2) relations reflecting social connectedness between nodes such as role-based (e.g. friends) and affective (e.g. likes) connections (3) interactions, and (4) flows such as intangible materials (e.g. beliefs, information) between interacting nodes in the network. SBNs also facilitate connecting with influential users (e.g. celebrities, experts, leaders) (Kane et al. 2014). Collectively, the above-mentioned features represent strong value propositions reflecting the identity of SBNs. Such features constitute a clear advantage that increases the likelihood of users on SBNs to continue their news consumption on SBNs. Thus, this study proposes:

Hypothesis 1a: Relative advantage of a social broadcasting network will lead to greater continuance of news consumption in the network.

The relative advantage of SBNs is likely to be less salient in relation to switching news consumption to traditional news mediums. This is because substitutable products (i.e. traditional news mediums such as television) have to prove their unique advantages over the incumbent product (i.e. SBNs) (Ye et al. 2008). However, if individuals expect other advantages or benefits from the substitutable product or service, then they are likely to switch to the substitutable product (Ye et al. 2008). As a ubiquitous news media, SBNs offer users a wide array of affordances (Majchrzak et al. 2013) and content selection (Lee and Ma 2012). In the present digital age, individuals are active users and processors of information (Ball-Rokeach and Jung 2009) and therefore likely to evaluate the potential benefits of an SBN based on their usage and experience, for further use (Lee and Ma 2012). SBNs have increased public debates and social discussions (Agarwal et al. 2008) resulting in widespread proliferation of its use (Kane et al. 2014). Based on the above, this study posits:

Hypothesis 1b: Relative advantage of a social broadcasting network will reduce switching to traditional news media for consuming news.

\subsection{User Influence}

As Glynn et al. (2012) posits, the prime motivation for joining digital social networks is often social. However, when exposed to constant information posted by friends, colleagues or acquaintances, users start following other users in the networks (Glynn et al. 2012). Diverse activities influence users in these networks to actively engage in the network, wherein they do not have to rely on (i) news flows controlled by traditional news firms, and (ii) news content determined by editors and news practitioners (Lee and Ma 2012). Users on SBNs are 
influenced by celebrities, experts and leaders, as that (i) increases the users' reputation in social and professional networks (Kane et al. 2014) (ii) provides indirect connections, which are not possible in offline environments, and (iii) keeps users informed of CELs personal, social and professional lives in real time. A study by Williamson et al. (2012) on young users' news activities on Facebook finds that users prefer personal news in the form of information posted by friends or through status updates and reporting issues and events in real time (e.g. Facebook status update - Raining heavy, homes likely flooded). A respondent in the study by Williamson et al. (2012) says, "I suppose from blogs and Facebook and all those sorts of things, I get personal news, that I find out from other people" (Williamson et al. 2012, p. 262). Based on the above, this study hypothesizes the relationship between user influence in SBNs and continuance of news consumption in the network as

Hypothesis 2a: User Influence in a social broadcasting network will lead to greater continuance of news consumption in the network.

The diverse goals of institutional users (e.g. business, political and non-commercial entities) on SBNs include creating buzz, advertising, marketing, delivering products and services, understanding customer sentiments and gauging product popularity and customer loyalty (Divol et al. 2012; Hong 2012). News firms, as institutional users engage SBNs for (i) actively disseminating news (Hong 2012) (ii) ensuring that news reaches the masses through diverse user networks (Hong 2012), and (iii) diverting individuals from SBNs to their traditional mediums by embedding click back links (Pew Research Center 2013). This has made SBNs viable sources of information and news (Glynn et al. 2012; Shi et al. 2014). Prior studies on news media have described its influence on society and public opinion as well as powerful behavioural, cognitive, emotional and attitudinal effects on news consumers. A study by Williamson et al. (2012) on the role of news media and young adults finds that people prefer several sources for news and still find printed newspapers having high information credibility. News firms also permit their employees (e.g. reporters) to use SBNs for officially sharing information on behalf of the company. They routinely hire social media editors to manage the production and delivery of news content on SBNs (Glynn et al. 2012). In doing so, they deliver news to mass audiences with the aim of influencing them to consume news on their company's news mediums.

Hypothesis $2 b$ : User Influence in a social broadcasting network will increase switching to traditional news media, for consuming news.

\subsection{Post-adoptive Impact of News Consumption}

Literature on IT post-adoptive behaviour postulate that during a period of utilitarian or functional technology use, individuals engaging in the reflective use of technology trigger "technology sense making" (Jasperson et al. 2005, p. 544). This is based on how individuals use the technology's attributes or features rather than their own habitual use of the technology (Bhattacherjee 2001; Jasperson et al. 2005; Sedera et al. 2017). Post adoptive behaviours leading to future use of IT are also derived from self-orchestrated learning experiences. These are based on IT features and the work systems in which they are situated, which potentially modify user cognitions on the future use of the technology (Jasperson et al. 2005). This happens through experimentation with IT features and applications (DeSanctis and Poole 1994; Lokuge and Sedera 2016) as well as by observing how others are using similar IT applications (Bandura 1986; Lokuge et al. 2016). In this study, it is observed that individuals reflecting switching and continuance news behaviours tend to rely less frequently in reflecting on other attributes of 
SBNs. Instead, they rely on their news consumption experience for future news consumption in an SBN. Thus, it is pragmatic to apply prior-use experiences to imprint future news consumption, which occur automatically in response to an urge for consuming news. According to Jasperson et al. (2005) past IT use behaviour generally produces a tendency for similar future actions in a given context, which dissipate reflective cognitive processing and result in more routinized behaviours. Based on the above, this study posits that news consumption of individuals on SBNs will depend on their existing news behaviours rather than user intentions or decisions. With respect to continuing news consumption in SBNs, this study hypothesizes that the habitual continuance of news consumption in these networks is a dominant predictor of a network supporting higher consumption of news compared to traditional news mediums.

Hypothesis 3: Continual consumption of news in a social broadcasting network will increase the duration of news consumed in the network compared to a traditional news medium.

Present-day technologies that cater to individual use are often highly substitutable products (or services) that have similar functionalities and compete with each other (e.g. web browsers) (Ye et al. 2008). Such settings induce users to terminate, reduce or concurrently use the incumbent IT/IS application and switch to functionally related products or services. However, such switching behaviours occur only in post-adoption IT/IS use settings where adoption, use and familiarity with the new product or service has taken place (Salim et al. 2014; Ye et al. 2008). A study by the Pew Research Centre relating to news on Facebook finds news consumers regularly watch news on television and read printed newspapers (Pew Research Center 2013). Thus, this study hypothesizes,

Hypothesis 4: Switching news consumption from a social broadcasting network to traditional news media will reduce the duration of news consumption in the network.

The influence of SBNs and the influence in SBNs form the antecedents to the news behaviours of switching and continuance. These behaviours are self-reported activities comprising news consumption on SBNs. The outcome of these behaviours is depicted through the comparative duration of news consumption on SBNs.

\section{Methodology}

This study employs the survey (qualitative) method. This is influenced by the study objectives, which seek to test and verify the relationship between individuals' news behaviours and the factors influencing those behaviours. The quantitative method is also used based on a careful review of related studies. For example, Bhattacherjee et al. (2012) use a survey to empirically test users switching from one IT product to another (contextualized to web browsers) in an environment of competitive technology choices. Park and Ryoo (2013) employ the survey method to empirically examine the relationship between two switching factors (viz. switching enablers and switching inhibitors) and intention of users to switch to cloud services. The quantitative method is effective in endorsing or challenging hypothetical relationships and uncovering new associations through statistical analysis and objective verifications (Danziger and Kraemer 1991; Gable 1994; Kling 1991). Past studies have also shown that the quantitative method (i) has superior deducibility over the qualitative (e.g. case studies) method (Vidich and Shapiro 1955; Walther et al. 2013b) (ii) can accurately document norms, identify outcomes and demarcate relationships between variables (Attewel and Rule 1991; Lokuge and Sedera 2014b; Vidich and Shapiro 1955) (iii) is a sound methodology for data verification (Gable 1994; 
Walther et al. 2013a), and (iv) facilitates data analyses at aggregate as well as individual levels thus offering better explanations about the validity of the survey instrument and the research model (Benbasat 1984; Sedera 2006).

This study employed a seven point Category scale for capturing the frequency (Zikmund 2000) of news consumption. The scale measures how individuals rate the extent of their news consumption activities. The categories on the scale move from the left (negative) to the right (positive) side of the scale. They are: Never, Rarely, Just Now \& Then, Sometimes, Often, Very often, All of the Time. Numbers from 1 (Never) to 7 (All of the Time) are assigned as anchors to each category for data analysis. The interval between each category is treated as equal. Thus, the scale can also be used as interval measurement scale (Cavana et al. 2001; Sedera et al. 2003). The category 'Sometimes' (the 4th in the 7-point scale) is taken as the midpoint of the scale and treated as neutral to the scale or a "passing level" (Refer to Cavana et al. 2001, p. 205) for rating the extent of news consumption. In comparison: (i) Often (the 5th in the 7-point scale) and points thereafter to its right (6th and 7th) show an ascending degree of frequency wherein the extent of news consumption is considered positive, and (ii) Just Now \& Then (the 3rd in the 7point scale) and points thereafter to its left (2nd and 1st) show a descending degree of frequency wherein the extent of news consumption is considered negative.

All questions in the survey were mandatory. The sole criterion for taking the survey was respondents had to be existing users of SBNs (e.g. Twitter, Facebook) and traditional news mediums (e.g. news websites, television or newspapers). In order to eliminate and reduce the influence of common method variance, the study employed different sources of information during measures development and assorted the order of questions to reduce the likelihood of theory-in-use bias (Chang et al. 2010). Further, this study ensured that survey questions were (i) appropriately worded to convey precise information, and (ii) shortened to reduce ambiguity, maintain clarity, and influence participation and response (Williams et al. 2003).

\subsection{Survey Deployment}

This study employed face validation procedures (similar to content validation) wherein the appropriateness of survey items for accuracy, language and appearance was evaluated by subjective judgements of experts (Keszei et al. 2010). It consulted a group of nine respondents comprising post-graduate students, doctoral researchers, academics and the public. The group provided constructive critique and feedback on (i) improving the construct definitions (ii) refining the construct measures and measurement items, and (iii) improving the measurement items. The feedback improved the item wordings, readability, meaning and content validity of the instrument (Schouten et al. 2010). The instrument was then pre-tested with 21 respondents (10 individuals responded to the survey online while 11 responded to the survey offline by completing a paper-based questionnaire). Next, the survey instrument was pilot tested with 40 respondents for assessing reliability and validity of individual items, which yielded acceptable results. See Appendix A.

The main survey was offered simultaneously in three ways: (1) a link to the online survey was emailed to students and staff at a tertiary institution (2) printed copies of the survey were handed out to students, at random, on campus at the institution, and (3) it was posted online on SBNs (e.g. Facebook and LinkedIn). The criteria for taking the survey was respondents had to be potential or existing news consumers on SBNs as well as traditional news mediums (e.g. TV). A total of 325 individuals successfully completed the survey (Note: the online survey recorded 969 clicks). Given the limitations of time and available resources, a small 
representative sample of the population had been pre-planned. In deploying quantitative methods, smaller groups are useful in making inferences about bigger groups that are potentially exorbitant to study (Barlett et al. 2001). The sample size comprising 325 respondents is deemed reasonable and representative of the population based on Cochran (1977) formula for determining basic sample size. In doing so, the alpha level a-priori was set at 0.05 , and based on the 7-point Category scale the level of acceptable error was set at $3 \%$ with standard deviation as 1.167 . The sample size is also adequate based on the number of items used in gathering data (Note: the survey instrument contains 23 items).

\section{Data Analysis and Findings}

\subsection{Reliability Tests}

Based on the work of Barclay et al. (1995), this study examined the construct reliability of the research model by assessing individual measurement item reliability, internal consistency and discriminant validity. Structural Equation Modelling (SEM) was employed for analysing the data using AMOS 22.0 and the measurement model was examined prior to assessing the structural (research) model (Hsieh and Wang 2007). The measurement attributes of all constructs were estimated with confirmatory factor analysis and items with low loadings were deleted. The resultant indices show an acceptable baseline fit. The RMSEA and CFI of the model is on par with the desired 0.08 and 0.90 levels respectively (Browne and Cudeck 1993). The research model indices relating to the Tucker-Lewis Index (TLI) (Hsieh and Wang 2007) is baseline with the desired criterion level. Next, this study examined the discriminant validity, internal consistency and convergent validity by evaluating the Cronbach's alpha, composite reliability (CR), and average variance extracted (AVE) of each construct. Cronbach's alpha and composite reliability values are consistent with the recommended 0.70 levels (Nunnally et al. 1967) while AVE values are acceptable, as they meet the desired 0.50 level (Fornell and Larcker 1981). Thus, convergent validity level is acceptable confirming that all items are consistent, reliable, and reflect the properties that the construct represents. Further, this study conducted the discriminant validity test to measure the difference between constructs in the research model. This was done through AMOS (version 22.0) by assessing the average value of each construct and comparing its squared correlations with other constructs. The analysed data also shows the average shared variable (ASV) and when compared to the average variance extracted (AVE), the ASV is less thereby offering strong support for discriminant validity of constructs (Fornell and Larcker 1981). (Note: Due to page limitations, internal consistency and convergent validity assessment, and comparisons of AVE and squared correlations have not been tabled in this paper)

\subsection{Research Model Testing}

The predictive power of standardised path coefficients, variance (R2) and path significance in the research model were examined using SPSS and its add-on module AMOS (version 22.0). The tested model using the sample data $(n=325)$ is illustrated in Figure 2. (Note: Due to page limitations, regression weights explaining variances between the constructs are not tabled in this paper). 


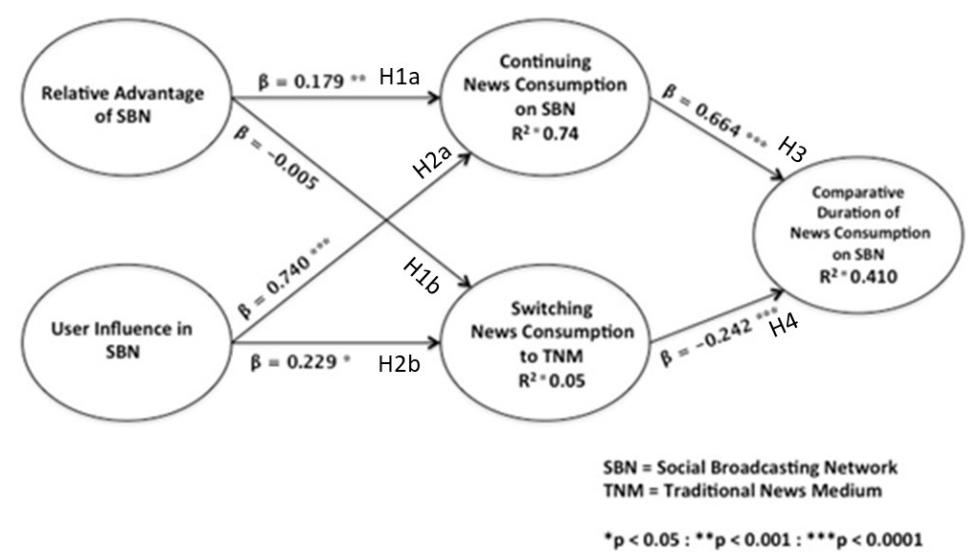

Figure 2. Tested Research Model ( $n=325)$

The examined sample ( $\mathrm{n}=325)$ reveals: (1) A significant path between user influence and continuing news consumption on SBNs. This shows that users in SBNs substantially sway other users in the network to consume news in the network (2) The relationship between user influence and switching news consumption to traditional news medium is significant. This implies that news practitioners and news firms (viz. institutional users) are successful in attracting news consumers to their contemporary news channels, although such relationships are potentially weak (3) The relative advantage of SBNs also shows a meaningful relationship with continuing news consumption in SBNs. This denotes that individuals consider the relative advantage of SBNs for seeking news on an immediate and timely basis and for connecting to both, information and users (4) The relative advantage of SBNs is not significant when individuals choose to switch their news consumption to traditional news mediums. This denotes that the relative advantage of SBNs is largely irrelevant or unimportant while deciding to switch to traditional news mediums for consuming news (5) Collectively, both constructs viz. user influence and relative advantage explain $74 \%$ of variance for continuing news consumption on SBNs while the same two constructs viz. user influence and relative advantage of SBNs demonstrate just 5\% variance in switching news consumption to traditional news mediums. This has serious implications for news firms that engage with SBNs for attracting people to their traditional news mediums, and (6) Collectively, switching and continuing news behaviours explain $41 \%$ variation in the duration of news consumption on SBNs compared to the duration of news consumption on traditional news mediums. This indicates that although people intermittently switch to traditional news mediums, the extent of such switching is low. Rather, most individuals consume news on SBNs on a continuing basis. Thus, although SBNs may benefit news firms by providing their platforms for delivering news, most people may not switch to traditional news mediums when they are on SBNs.

\subsection{Hypothesis Testing}

\subsubsection{Hypothesis $1 \mathrm{a}$}

Testing confirms that continuance of news consumption on SBNs increases when individuals evaluate the value propositions of SBNs for consuming news. The correlation between relative advantage and continuing news consumption is significant and shows positive path coefficient $(\beta=0.179$ with $p<0.001)$. The features and value propositions of SBNs represent a strong identity of the networks for consuming news. The data shows that individuals consider 
these more significant when making a media choice between SBNs and traditional news mediums. Therefore, the relative advantage of SBNs is a given fact, which does not require significant independent consideration when choosing a media for consuming news. The data supports Hypothesis 1a, "Relative advantage of a social broadcasting network will lead to greater continuance of news consumption in the network."

\subsubsection{Hypothesis $1 \mathrm{~b}$}

Testing shows a negative path coefficient between relative advantage of SBNs and switching news consumption to traditional news mediums. This relationship is evidenced as being not significant $(\beta=-0.005)$. As hypothesized, substitutable products such as traditional news mediums must prove their unique advantages or strengths over incumbent products such as SBNs to attract usage (Note: This study situates individuals already engaging with SBNs). Therefore, individuals are likely to switch to competitive products only if the expected benefits are greater than the incumbent products. However, the relative advantage of SBNs is not an important consideration for users wishing to switch to traditional news mediums for consuming news. Although features and value propositions of SBNs represent a strong identity of the networks for consuming news, users (specifically younger generations such as New Millennials) do not consider those as significant when choosing between SBNs and traditional news mediums. This is because they are already actively engaged in consuming information beyond news in SBNs (e.g. emailing, chat, games etc.). Thus, there is little resistance to adopting and using SBNs. So, to them, the relative advantage of SBNs is a given fact, which does not require significant and independent considerations if switching to other or traditional news mediums. In addition, our study also observed that news switching may not involve the user in completely abandoning the existing use of digital news artefacts or services but rather concurrently using complementing (yet competing news mediums) services. For example, an individual on Twitter may simultaneously watch a news channel on television while surfing the same news on Twitter. In such scenarios, switching (i.e. the complementing use of traditional news mediums) is likely to make the relative advantage of SBNs redundant.

Therefore, the data does not support Hypothesis $1 \mathrm{~b}$, "Relative advantage of a social broadcasting network will reduce switching to traditional news media, for consuming news."

\subsubsection{Hypothesis $2 a$}

The tested model shows a highly significant path coefficient between user influence in an SBN and continuing news consumption in the network. It shows a positive path coefficient $\beta=0.740$ with $\mathrm{p}<0.0001$. This is a significant finding that evidences the influences exerted by users in SBNs on other individuals in the network, which strongly contributes towards users continuing their news consumption on SBNs. This denotes that users do not switch over to traditional news mediums as a result thereof. Thus, findings concur with Hypothesis $2 \mathrm{a}$, "User Influence in a social broadcasting network will lead to greater continuance of news consumption in the network."

\subsubsection{Hypothesis $2 b$}

The tested model shows positive support but differing levels of significance between users influencing other users in SBNs and users switching their news consumption to traditional news mediums. The sample show a positive and significant path coefficient with $\beta=0.229$ with $p<0.05$. This is suggestive of a significant effect that institutional users such as news firms can 
have on individuals in SBNs. The evidence of switching news consumption to traditional news mediums happening simultaneously with continuing news consumption on SBNs suggests that SBNs have a dual effect on individuals in the network. However, compared to hypothesis $2 \mathrm{a}$, user influence on switching is lower than on continuing news consumption on SBNs. Findings support hypothesis $2 \mathrm{~b}$, "User Influence in a social broadcasting network will increase switching to traditional news media, for consuming news."

\subsubsection{Hypothesis 3}

The tested model finds the outcome of continuing news consumption on SBNs to higher duration of news consumption in the network to be highly significant and shows positive support for hypothesis 3, "Continually consuming news in a social broadcasting network will increase the duration of news consumed in the network compared to a traditional news medium." Findings evidence that continuance of news consumption on SBNs is a leading forecaster of the networks' supporting higher duration of news consumption compared to traditional news mediums. This has strong implications for news firms. It suggests that $\mathrm{SBN}$ s are superior news providers compared to traditional news firms.

\subsubsection{Hypothesis 4}

Findings show that when users switch from SBNs to traditional news mediums, it decreases their overall duration of news consumption on SBNs. Thus, switching to traditional news mediums has a negative impact on the overall duration of news consumption in SBNs. This supports hypothesis 4, "Switching news consumption from a social broadcasting network to traditional news media will reduce the duration of news consumption in the network."

\subsection{Key Findings}

The empirically validated research model depicts that SBNs influence individual news consumption showing greater duration of individual news consumption on the networks compared to traditional news mediums. Yet, it also shows support for individuals switching their news consumption to traditional news mediums. Summarized findings are: (1) Users in SBNs show a dual way of consuming news. On the one hand, they sometimes switch to traditional news mediums for consuming news while on the other they sometimes continue their news consumption in SBNs. Overall, users often spend more time in consuming news in SBNs compared to traditional news mediums (2) The antecedents to news switching and continuance, which is the relative advantage of SBNs has a significant impact on continuing news consumption on the networks. It has a negative impact on switching news consumption to traditional news mediums, which is not significant. User influence in SBNs has a highly significant impact on individuals continuing news consumption on SBNs while its impact on switching to traditional news mediums is found to be significant (3) Individuals switch to traditional news mediums only for specific information or for verifying information but overall continue consuming news for longer durations on SBNs. This indicates strong support for SBNs as news providers (4) News firms as institutional users deliver news through SBNs and potentially influence individuals to consume news through their traditional platforms as evidenced by the switching behaviour. However, higher duration of news consumption on SBNs (compared to traditional news mediums) denote that any benefits to news firms accrued because of switching news consumption is perhaps temporary and unsustainable in the long run (5) The data shows elevated levels of switching news consumption to traditional news mediums compared to continuing news consumption on SBNs. This is perhaps due to the exposure to both, traditional and digital media in equal measure. However, the duration of 
news consumption on SBNs shows an increasing trend indicating their potential superiority as news providers (6) The data shows a general decline in switching news consumption to traditional news mediums, and (7) User influence is evidenced as a key variable having a highly significant effect on individuals switching and continuing their news consumption on SBNs. Although social influence has been investigated by past studies, actual user influence has largely been under researched. So, this study clarifies and empirically validates the nuanced role of users in digital social networks.

Note: This study also analysed data relating to demographic features of respondents using descriptive statistics. The analysis featured sample demographic statistics classified through respondent's age, gender and geographical location. The data represents four applicable generation cohorts, viz. Baby Boomers (born before 1964), Generation X (1965-1978), Generation Y (1979-1989), and New Millennials (1990 and resulting thereafter). The analysed data showed $47.38 \%$ of news consumers on SBNs to be New Millennials (154 respondents), which comprises the biggest cohort amongst all groups, followed by Generation Y (24.31\%), Generation X (22.15\%) and Baby Boomers $(6 \%)$. The analysis depicted the increasing use of SBNs by successive generations. The male to female gender ratio was reasonably balanced in all groups with the New Millennials showing the biggest gap with $29.23 \%$ males compared to $18.25 \%$ females using SBNs for consuming news. Post-Hoc testing was conducted on the sample $(\mathrm{n}=325)$, which was polarized into two groups: (1) Baby Boomers + Generation $X+$ Generation Y, and (2) New Millennials.

\section{Conclusion}

Social broadcasting networks such as Facebook and Twitter (SBNs) (Shi et al. 2014) have infused the social element in the production and dissemination of news by giving users of such networks the power to report, share and consume information. Such information does not constitute news as understood in the traditional sense. Rather, they represent bits and pieces of information relating to incidences (e.g. events, actions) that occur on a 24/7 basis. This study investigated the news behaviours of switching and continuance on SBNs for explaining how they (i.e. SBNs) transform news consumption. It specifically examined the simultaneous switching and continuance of news consumption of 325 respondents in a post-adoption technology setting, i.e. individuals already engaging with SBNs for consuming news. It explained news behaviours through the notion of social homogeneity shaped by the environment of SBNs and empirically validated it through the constructs of (i) relative advantage of SBNs, as influence of the network, and (ii) user influence on other users as the influence in the network environment. Thus, by testing a model of news behaviours on SBNs, this study brings new understanding on the role of digital news media, communications and digital social networks, and the dependency relations between network environments and users.

\section{Contributions to Research and Implications for Practice}

Past studies have investigated news consumption on traditional news mediums and the Internet through broad themes such as (i) forming news repertoires across multiple media platforms (Yuan 2011) (ii) information overload and sense making (Pentina and Tarafdar 2014), and (iii) displacement effects of media (Lee and Leung 2008). However, those studies do not (i) define news consumption and consumption activities on any specific news medium 
(ii) investigate news consumption on a specific sub-class of digital social networks such as SBNs (iii) explain news behaviours such as switching and continuance (iv) examine the environmental influences of and in specific news mediums on news behaviours, and (v) highlight the role of digital social networks based on the empirical validation of news behaviours, all of which this study accomplishes. By adapting the Two Factor theory, this study expands the body of knowledge to a new sub-class of digital social technologies called SBNs (Shi et al. 2014). It explains how information is consumed at the individual level in SBNs due to the influence of SBNs and the influence in SBNs. Focusing on the domain of news media it builds and empirically validates a research model depicting news consumption on SBNs.

First, it directs the attention of researchers towards distinguishing the role of digital social technologies such as Facebook or Twitter as SBNs. It clarifies the role of Facebook, Twitter and similar technologies as information broadcasters by highlighting their social and broadcasting features by comparing them with traditional news mediums. Such evaluations facilitate understanding the epistemic value of SBNs as news providers. There is disagreement on the effectiveness of digital social technologies in enabling citizen journalism and taking up the role of gatekeeper in informing society and enhancing civic knowledge (Pentina and Tarafdar 2014). However, this study reveals a more nuanced role of SBNs, through the news behaviours of individuals. It demonstrates the potential benefits to news firms because of individuals switching from SBNs to traditional news mediums while in the same breadth highlights the potential of SBNs becoming competitive news providers. This is perhaps one of the first studies in IS providing empirical evidence on individual news consumption behaviours on a digital social network, which leads the study (future) towards interpreting the role of technology (i.e. SBNs) at the organisational level.

Second, IT switching is an emerging yet under-researched area in IS (Bhattacherjee et al. 2012) while IT continuance has attracted relatively more attention as a set of affective responses to IT acceptance and use (Kim and Malhotra 2005). This study concisely demonstrates the integration of both, switching and continuance for explaining news consumption. It positions switching and continuance as post-IT adoption behaviours and explains actual news consumption rather than intentions.

Third, in addressing the causes of IT continuance behaviours, this study takes a more recursive stance in positioning switching and continuance as two opposing news consumption behaviours. It adapts the theoretical lens of the Two-Factor theory and develops the constructs of relative advantage (signifying influence of SBNs) and user influence (indicating influence in the networks) as two factors for explaining why users switch or continue consuming news on SBNs. It explains new dynamics of intermittent or alternate use of SBNs, which has not been investigated by prior studies. Also, it demonstrates that when online and offline news mediums co-exist, influence of users on other users in the incumbent (online) medium plays a key role in inducing the decision to use or refrain from using a substitutable offline medium. Relative advantage has been described in past studies as the best predictor of IT adoption leading to actual usage (Karahanna et al. 1999) as well as in IT switching intentions (Bhattacherjee et al. 2012). However, this study finds that it does not have a significant effect on users deciding to either switch news consumption to traditional news mediums or continue news consumption on SBNs. This study tests the two-factor theory in a post-adoption setting on two opposing news behaviours of switching and continuance within the context of social digital technologies, which has not been attempted by past studies in IS. 
Fourth, findings contribute to the IS, media and communications domains by constructing and validating a model of news behaviours through two key factors (viz. relative advantage and user influence). In general, this study systemically assesses unique or potentially sensitive effects of a specific sub-class of digital social technologies (i.e. SBNs) at the individual level.

Fifth, in recent times there has been much debate (especially in the U.S. in the aftermath of the 2017 presidential elections) on whether contemporary media manipulates news (popularly termed as fake news). This study provides a conceptual guide (Refer to Figure 1) into how consumers can alternate between traditional news mediums and SBNs for newsworthy information. This implies that news consumers have a choice of selecting traditional news channels that purportedly propagate fake news, or consume news aggregated, curated or broadcast at grassroot levels by people using SBNs. Such participatory, social and partisan nature of news reporting suggests (as per our preliminary observations) that consumers are likely to be persuaded by family and close friends, and people of repute such as entertainers, business and political leaders. However, in recent times even SBNs have been plagued (e.g. YouTube, Twitter) by hoaxes peddling false and misleading information created by unverified channels. So, although user influence impacts news consumption behaviours (especially continuity on SBNs, as evidenced by our study) people and channels masquerading as newsworthy outlets make it difficult to filter contextual expression and validity of information flows from genuine news reporting This phenomenon requires further and in-depth attention and research.

\subsection{Implications for Practice}

This study evidences that SBNs are no longer static channels for information flows between senders and receivers. Rather, they have become veritable repositories enabling: (i) dynamic interactivity in real time between mass communicators (ii) reporting, sharing and creating diverse content and views by mass users (iii) selectivity and control over information and usage of the network (iv) personalization of content (v) convergence of technological boundaries between communication forms and social elements, and (vi) global reach and media portability (Metzger, 2009). Such strategic choices that exploit or explore the opportunities offered SBN are important determinants of firm survival. News consumption on SBNs shows that news firms engage with SBNs as institutional users to complement their business function of news delivery. This can happen due to major shifts within the news firm in relation to (i) limitations of traditional business models, as any modern technology that enhances readership is more than likely to limit the firm's incumbent business model including its scope and scale, making it imperative for the firm to adopt the technology and fuse it with existing products and processes (ii) limited trans-functional role of IT: For example, the function of a television news-broadcasting firm requires a different technology (i.e. broadcasting), which is different from the technology required for creating content (i.e. publishing and editing system), and (iii) increased familiarity with IT: SBNs are easy to use. Their client-interface and usability is intuitively designed for use and is less cumbersome than the applications of legacy systems. However, given the low cost of engagement and no requirements for discontinuing existing products, services, functions and processes, news firms need to be vigilant in engaging with SBNs for delivering news to mass audiences in a bid to attract them to their news mediums. This implies that although SBNs provide their platforms for all users, and never meant to compete with news firm, users in an SBN influence other users and in the process, build the potential competing power of the network as a superior news provider. Hence, news firms need to provide more contextual information or 
in-depth news coverage and analysis for ensuring longer news consumption on their traditional mediums. This can potentially strengthen their subscription-based revenues for trustworthy and premium news content in the face of rising unverifiable information by online user-generated reports. The powerful significance of user influence, as found by this study can be applied to other domains of information consumption. For example, peer influence or biased product reviews can intensify or demystify the information asymmetry of individuals and limit (or increase) their exposure and choice to new product information and pricing. Behavioural economics proposes that people are generally affected by a paradox of choice, in which increasing number of options increases the difficulty of decision-making. So, information from familiar or authoritative sources is perceived as having greater value and significance in the decision-making process. Through this study we observe that users (i) are influenced by who (e.g. other users) communicates newsworthy information (ii) are motivated by what (they think) other users do (e.g. commenting, posting on SBNs) (iii) are influenced by user cues (e.g. like's) that shape actions, and (iv) act and feel better about what they do based on emotional attachments or applause from other users. Therefore, organizations (e.g. hotels, airlines) need to evaluate and manage ways in which patrons and consumers provide comments and/or information relating to the organization's products and services as well as their (i.e. consumers') experiences. Curating and sharing product information through personal blogs can negatively (or positively) impact the buying decisions of consumers. This study can be further extended by three academic domains viz. journalism, communications, and management. SBNs and consumption behaviours can be analysed at three levels: (1) individual users and society (public), (2) intermediaries and platforms, and (3) organisations and industries.

\subsection{Limitations}

The generalized findings are limited by the data sample size and the chosen news media. Both, SBNs and traditional news mediums are freely available and constitute reasonably strong substitutes. Users can voluntarily choose one over the other. Thus, further research is required for identifying and evaluating more salient factors other than those developed by this study. A bigger data sample size is also proposed (future research) for improving generalized findings. SBNs are not conceptualised by this study as monolithic IT artefacts. Rather, they represent a collection of many mediums, platforms and applications that have varied capabilities (e.g. Twitter is not the same as Facebook). Therefore, it is possible that the generic attributes of different SBNs may not be homogenous across all SBNs. So, the reflective role of SBNs may differ across networks. In addition, choosing news mediums exhibiting strong disparities in relative advantage can reveal stronger effects on switching and continuance behaviour than the findings reported by this study. The research model and the interpretations of findings are situated in a post-IT adoption setting pertaining to personal use.

\section{References}

Agarwal, R., Gupta, A. K., and Kraut, R. 2008. "Editorial Overview - the Interplay between Digital and Social Networks," Information Systems Research (19:3), pp. 243-252.

Alarifi, A., and Sedera, D. 2014. "Peripheral, Central and Coercive Routes for Promoting Enterprise Social Networks," Australasian Conference on Information, Auckland, New Zealand: ACIS. 
Alarifi, A., Sedera, D., and Recker, J. 2015. "Posters Versus Lurkers: Improving Participation in Enterprise Social Networks through Promotional Messages," in: International Conference on Information Systems. Fort Worth, Texas, USA: AIS.

Attewel, P., and Rule, J. B. (eds.). 1991. Survey and Other Methodologies Applied to IT Impact Research: Experiences from a Comparative Study of Business Computing. Boston, MA: Harvard Business School Press.

Ball-Rokeach, S. J., and Jung, J.-Y. 2009. "The Evolution of Media System Dependency Theory," in The Sage Handbook of Media Processes and Effects, R.L. Nabi and M.B. Oliver (eds.). Thousand Oaks, California: SAGE Publications, Inc., pp. 531-544.

Bandura, A. 1986. Social Foundations of Thought and Action: A Social Cognitive Theory. PrenticeHall, Inc.

Barclay, D., Higgins, C., and Thompson, R. 1995. "The Partial Least Squares (PLS) Approach to Causal Modeling: Personal Computer Adoption and Use as an Illustration," Technology Studies (2:2), pp. 285-309.

Barlett, J. E., Kotrlik, J. W., and Higgins, C. C. 2001. "Organizational Research: Determining Appropriate Sample Size in Survey Research," Information Technology, Learning, and Performance Journal (19:1), p. 43.

Benbasat, I. (ed.) 1984. An Analysis of Research Methodologies. Boston, M.A: Harvard Business School Press.

Bhattacherjee, A. 2001. "Understanding Information Systems Continuance: An ExpectationConfirmation Model," MIS Quarterly (25:3), pp. 351-370.

Bhattacherjee, A., Limayem, M., and Cheung, C. M. K. 2012. "User Switching of Information Technology: A Theoretical Synthesis and Empirical Test," Information \& Management (49:7-8), pp. 327-333.

Borgatti, S. P., and Foster, P. C. 2003. "The Network Paradigm in Organizational Research: A Review and Typology," Journal of Management (29:6), pp. 991-1013.

Borgatti, S. P., Mehra, A., Brass, D. J., and Labianca, G. 2009. "Network Analysis in the Social Sciences," Science (323:5916), pp. 892-895.

Browne, M. W., and Cudeck, R. 1993. Alternative Ways of Assessing Model Fit. Thousand Oaks, CA.: Sage.

Cavana, R. Y., Delahaye, B. L., and Sekaran, U. 2001. Applied Business Research: Qualitative and Quantitative Methods. Milton, Queensland: John Wiley \& Sons Australia, Ltd.

Cenfetelli, R. T., and Schwarz, A. 2010. "Identifying and Testing the Inhibitors of Technology Usage Intentions," Information Systems Research (22:4), pp. 808-823.

Chang, S.-J., van Witteloostuijn, A., and Eden, L. 2010. "From the Editors: Common Method Variance in International Business Research," J Int Bus Stud (41:2), pp. 178-184.

Choudhury, V., and Karahanna, E. 2008. "The Relative Advantage of Electronic Channels: A Multidimensional View," MIS Quarterly (32:1), pp. 179-200.

Cochran, W. 1977. Sampling Techniques. New York: Wiley and Sons. 
Coleman, J. S. 1990. Foundations of Social Theory. Cambridge, Mass: Belknap Press of Harvard University Press.

Cooper, R. B., and Zmud, R. W. 1990. "Information Technology Implementation Research: A Technological Diffusion Approach," Management Science (36:2), pp. 123-139.

Danziger, J. N., and Kraemer, K. L. (eds.). 1991. Survey Research and Multiple Operationalism. The Urbis Project Methodology. Boston, MA: Harvard Business School Press.

Davis, F. D. 1989. "Perceived Usefulness, Perceived Ease of Use and User Acceptance of Information Technology," MIS Quarterly (13:3), pp. 319-340.

DeSanctis, G., and Poole, M. S. 1994. "Capturing the Complexity in Advanced Technology Use: Adaptive Structuration Theory," Organization Science (5:2), pp. 121-147.

Divol, R., Edelman, D., and Sarrazin, H. 2012. "Demystifying Social Media," in: McKinsey Quarterly. McKinsey \& Company.

Fornell, C., and Larcker, D. F. 1981. "Evaluating Structural Equation Models with Unobservable Variables and Measurement Error," Journal of Marketing Research (18:1), pp. 39-50.

Gable, G. G. 1994. "Integrating Case Study and Survey Research Methods: An Example in Information Systems," European Journal of Information Systems (3:2), pp. 112-126.

Glynn, C. J., Huge, M. E., and Hoffman, L. H. 2012. "All the News That's Fit to Post: A Profile of News Use on Social Networking Sites," Computers in Human Behavior (28:1), pp. 113119.

Hanna, R., Rohm, A., and Crittenden, V. L. 2011. "We're All Connected: The Power of the Social Media Ecosystem," Business Horizons (54:3), pp. 265-273.

Herzberg, F., Mausner, B., and Snyderman, B. 1959. The Motivation to Work. New York: Wiley.

Hirst, M. 2011. News 2.0: Can Journalism Survive the Internet? Crows Nest, NSW 2065, Australia: Allen \& Unwin.

Hogg, M. A., and Turner, J. C. 1985. "Interpersonal Attraction, Social Identification and Psychological Group Formation," European Journal of Social Psychology (15:1), pp. 51-66.

Hong, S. 2012. "Online News on Twitter: Newspaper's Social Media Adoption and Their Online Readership," Information Economics and Policy (24:1), pp. 69-74.

Hsieh, P.-A. J. J., and Wang, W. 2007. "Explaining Employees' Extended Use of Complex Information Systems," European Journal of Information Systems (16), pp. 216-227.

Hsu, C.-L., Yu, C.-C., and Wu, C.-C. 2014. "Exploring the Continuance Intention of Social Networking Websites: An Empirical Research," Information Systems and e-Business Management (12:2), pp. 139-163.

Huang, E. 2009. "The Causes of Youths' Low News Consumption and Strategies for Making Youths Happy News Consumers," Convergence: The International Journal of Research into New Media Technologies (15:1), pp. 105-122.

Hulin, C. L., and Smith, P. A. 1967. "An Empirical Investigation of Two Implications of the Two-Factor Theory of Job Satisfaction," Journal of Applied Psychology (51:5, Pt.1), pp. 396402. 
Jasperson, J. S., Carter, P. E., and Zmud, R. W. 2005. "A Comprehensive Conceptualization of Post-Adoptive Behaviors Associated with Information Technology Enabled Work Systems," MIS Quarterly (29:3), pp. 525-557.

Kane, G. C., Alavi, M., Labianca, G., and Borgatti, S. P. 2014. "What's Different About Social Media Networks? A Framework and Research Agenda," MIS Quarterly (38:1), pp. 275304.

Kang, J., and Lee, H. 2017. "Modeling User Interest in Social Media Using News Media and Wikipedia," Information Systems (65), pp. 52-64.

Kaplan, A. M., and Haenlein, M. 2012. "Social Media: Back to the Roots and Back to the Future," Journal of Systems and Information Technology (14:2), pp. 101-104.

Karahanna, E., Straub, D. W., and Chervany, N. L. 1999. "Information Technology Adoption across Time: A Cross-Sectional Comparison Pf Pre-Adoption and Post-Adoption Beliefs," MIS Quarterly (23:2), pp. 183-213.

Keszei, A. P., Novak, M., and Streiner, D. L. 2010. "Introduction to Health Measurement Scales," Journal of Psychosomatic Research (68:4), pp. 319-323.

Kietzmann, J. H., Hermkens, K., McCarthy, I. P., and S., S. B. 2011. "Social Media? Get Serious! Understanding the Functional Building Blocks of Social Media," Business Horizons (54), pp. 241-251.

Kim, S. S., and Malhotra, N. K. 2005. "A Longitudinal Model of Continued Is Use: An Integrative View of Four Mechanisms Underlying Postadoption Phenomena," Management Science (51:5), pp. 741-755.

Kling, R. (ed.) 1991. Adapting Survey Methods to Study the Social Consequences of Computerization: A Response to Attewel and Rule Based on Five Survey-Based Studies. Boston, MA: Harvard Business School Press.

Krums, J. j. 2009. "Http://Twitpic.Com/135xa - There's a Plane in the Hudson. I'm on the Ferry Going to Pick up the People. Crazy. [Tweet]." https://twitter.com/jkrums/status/1121915133.

Kwak, H., Lee, C., Park, H., and Moon, S. 2010. "What Is Twitter, a Social Network or a News Media?," in: World Wide Web 2010. Raleigh, North Carolina, USA.: International World Wide Web Conference Committee (IW3C2), pp. 591-600.

Latané, B. 1981. "The Psychology of Social Impact," American Psychologist (36:4), pp. 343-356.

Lee, C. S., and Ma, L. 2012. "News Sharing in Social Media: The Effect of Gratifications and Prior Experience," Computers in Human Behavior (28:2), pp. 331-339.

Lee, P. S. N., and Leung, L. 2008. "Assessing the Displacement Effects of the Internet," Telematics and Information (25:25), pp. 145-155.

Lee, S., Shin, B., and Lee, H. G. 2009. "Understanding Post-Adoption Usage of Mobile Data Services: The Role of Supplier-Side Variables," Journal of the Association for Information Systems (10:12), pp. 860-888.

Li, H. L., and Ng, V. T. Y. 2013. "Discovering Associations between News and Contents in Social Network Sites with the D-Miner Service Framework," Journal of Network and Computer Applications (36:6), pp. 1651-1659. 
Lin, T.-C., and Huang, S.-L. 2014. "Understanding the Determinants of Consumers' Switching Intentions in a Standards War," International Journal of Electronic Commerce (19:1), pp. 163189.

Liu, C.-T., Guo, Y. M., and Lee, C.-H. 2011. "The Effects of Relationship Quality and Switching Barriers on Customer Loyalty," International Journal of Information Management (31:1), pp. 71-79.

Lokuge, S., and Sedera, D. 2014a. "Enterprise Systems Lifecycle-Wide Innovation," Americas Conference on Information Systems (AMCIS 2014), Savannah, Georgia: AIS.

Lokuge, S., and Sedera, D. 2014b. "Enterprise Systems Lifecycle-Wide Innovation Readiness," Pacific Asia Conference on Information Systems (PACIS 2014), Chengdu, China: AIS.

Lokuge, S., and Sedera, D. 2016. "Is Your IT Eco-System Ready to Facilitate Organizational Innovation? Deriving an IT Eco-System Readiness Measurement Model," The International Conference on Information Systems (ICIS2016), Dublin, Ireland: AIS.

Lokuge, S., Sedera, D., and Grover, V. 2016. "Thinking inside the Box: Five Organizational Strategies Enabled through Information Systems," Pacific Asia Conference on Information Systems (PACIS 2016), Chiyai, Taiwan: AIS.

Maddox, R. N. 1981. "Two-Factor Theory and Consumer Satisfaction: Replication and Extension," Journal of Consumer Research (8:1), pp. 97-102.

Majchrzak, A., Faraj, S., Kane, G. C., and Azad, B. 2013. "The Contradictory Influence of Social Media Affordances on Online Communal Knowledge Sharing," Journal of ComputerMediated Communication (19:1), pp. 38-55.

Nunnally, J. C., Bernstein, I. H., and Berge, J. M. T. 1967. Psychometric Theory. New York: McGraw-Hill.

Ortiz de Guinea, A., and Markus, M. L. 2009. "Why Break the Habit of a Lifetime? Rethinking the Roles of Intention, Habit, and Emotion in Continuing Information Technology Use," MIS Quarterly (33:3), pp. 433-444.

Palekar, S., Atapattu, M., Sedera, D., and Lokuge, S. 2015. "Exploring Spiral of Silence in Digital Social Networking Spaces," The International Conference on Information Systems (ICIS 2015), Fort Worth, Texas: AIS.

Park, S. C., and Ryoo, S. Y. 2013. "An Empirical Investigation of End-Users' Switching toward Cloud Computing: A Two Factor Theory Perspective," Computers in Human Behavior (29:1), pp. 160-170.

Pentina, I., and Tarafdar, M. 2014. "From "Information" to "Knowing": Exploring the Role of Social Media in Contemporary News Consumption," Computers in Human Behavior (35), pp. 211-223.

Pew Research Center. 2013. The Role of News on Facebook: Common yet Incidental, Pew Research Center, Washington D.C, United States, pp. 1-24.

Ren, Y., Harper, F. M., Drenner, S., Terveen, L., Kiesler, S., Reidl, J., and Kraut, R. E. 2012. "Building Member Attachment in Online Communities: Applying Theories of Group Identity and Interpersonal Bonds," MIS Quarterly (36:3), pp. 841-864.

Rogers, E. M. 1995. Diffussion of Innovations, (4th ed.). New York: The Free Press. 
Salim, S. A., Sedera, D., Sawang, S., and Alarifi, A. 2014. "Technology Adoption as a MultiStage Process," Australasian Conference on Information Systems (ACIS), Auckland, New Zealand: ACIS.

Schouten, L., Grol, R., and Hulscher, M. 2010. "Factors Influencing Success in QualityImprovement Collaboratives: Development and Psychometric Testing of an Instrument," Implementation Science (5:1), p. 84.

Sedera, D. 2006. "Enterprise Systems Success Measurement Model," in: School of Information Systems. Brisbane: Queensland University of Technology, p. 456.

Sedera, D., Gable, G., and Chan, T. 2003. "Survey Design: Insights from a Public Sector-ERP Impact Study," Proceedings of the $7^{\text {th }}$ Pacific Asia Conference on Information Systems, J. Hanisch, D. Falconer, S. Horrocks and M. Hillier (eds.), Adelaide, Australia: Association for Information Systems, pp. 595-610.

Sedera, D., Lokuge, S., Atapattu, M., and Gretzel, U. 2017. "Likes-the Key to My Happiness: The Moderating Effect of Social Influence on Travel Experience," Information $\mathcal{E}$ Management (54:6), pp. 825-836.

Sedera, D., Lokuge, S., Grover, V., Sarker, S., and Sarker, S. 2016a. "Innovating with Enterprise Systems and Digital Platforms: A Contingent Resource-Based Theory View," Information $\mathcal{E}$ Management (53:3), pp. 366-379.

Sedera, D., Lokuge, S., Salleh, N., Moghavvemi, S., and Palekar, S. 2016b. "Spoilt for Choice: When User-System Relationship Becomes One-to-Many," The International Conference on Information Systems (ICIS2016), Dublin, Ireland: AIS.

Setia, P., Venkatesh, V., and Joglekar, S. 2013. "Leveraging Digital Technologies: How Information Quality Leads to Localized Capabilities and Customer Service Performance," MIS Quarterly (37:2), pp. 565-A564.

Shi, Z., Rui, H., and Whinston, A. B. 2014. "Content Sharing in a Social Broadcasting Environment: Evidence from Twitter," MIS Quarterly (38:1), pp. 123-142.

Velichety, S., and Ram, S. 2013. "A Cross-Sectional and Temporal Analysis of Information Consumption on Twitter," Thirty Fourth International Conference on Information Systems, Milan, Italy.

Venkatesh, V., and Davis, F. D. 2000. "A Theoretical Extension of the Technology Acceptance Model: Four Longitudinal Field Studies," Management Science (45:2), pp. 186-204.

Venkatesh, V., Thong, J. Y. L., and Xu, X. 2012. "Consumer Acceptance and Use of Information Technology: Extending the Unified Theory of Acceptance and Use of Technology," MIS Quarterly (36:1), pp. 157-178.

Vidich, A. J., and Shapiro, G. 1955. "A Comparison of Participant Observation and Survey Data," American Sociological Review (20:1), pp. 28-33.

Walther, S., Sarker, S., Sedera, D., and Eymann, T. 2013a. "Exploring Subscription Renewal Intention of Operational Cloud Enterprise Systems-a Socio-Technical Approach," European Conference on Information Systems, Utrecht, The Netherlands: AIS, p. 25. 
Walther, S., Sedera, D., Sarker, S., and Eymann, T. 2013b. "Evaluating Operational Cloud Enterprise System Success: An Organizational Perspective," European Conference on Information Systems (ECIS 2013), Utrecht, p. 16.

Wasko, M. M., and Faraj, S. 2005. "Why Should I Share? Examining Social Capital and Knowledge Contribution in Electronic Networks of Practice," MIS Quarterly (29:1), pp. 35-57.

Williams, L. J., Edwards, J. R., and Vandenberg, R. J. 2003. "Recent Advances in Causal Modeling Methods for Organizational and Management Research," Journal of Management (29:6), pp. 903-936.

Williamson, K., Qayyum, A., Hider, P., and Liu, Y.-H. 2012. "Young Adults and Everyday-Life Information: The Role of News Media," Library \& Information Science Research (34:4), pp. 258-264.

Wu, S., Hofman, J. M., Mason, W. A., and Watts, D. J. 2011. "Who Says What to Whom on Twitter," World Wide Web, Hyderabad, India: International World Wide Web Conference Committee (IW3C2), pp. 705-714.

Xu, C., Ryan, S., Prybutok, V., and Wen, C. 2012. "It Is Not for Fun: An Examination of Social Network Site Usage," Information \& Management (49), pp. 210-217.

Ye, C., Seo, D., Desouza, K. C., Sangareddy, S. P., and Jha, S. 2008. "Influences of IT Substitutes and User Experience on Post-Adoption User Switching: An Empirical Investigation," Journal of the American Society for Information Science and Technology (59:13), pp. 2115-2132.

Yuan, E. 2011. "News Consumption across Multiple Media Platforms," Information, Communication \& Society (14:7), pp. 998-1016.

Zikmund, W. G. 2000. Business Research Methods (Sixth Edition). Orlando, Florida, USA: The Dryden Press.

Zmud, R. W. 1982. "Diffusion of Modern Software Practices: Influence of Centralization and Formalization," Management science (28:12), pp. 1421-1431. 


\section{Appendix A}

\section{DEMOGRAPHICS}

\section{Gender}

○ Female

$\odot$ Male

\section{Age}

I was born

๑ Before 1964

$\odot$ Between 1965 and 1978

๑ Between 1979 and 1989

$\odot 1990$ or after

\section{Location}

I live in

$\odot$ Australia / New Zealand

$\odot$ North America / Canada

$\odot$ Europe

$\odot$ Middle East

$\odot$ South East Asia

$\odot$ China / North East Asia

$\odot$ Indian sub-continent / Sri Lanka

$\odot$ Africa

PLEASE INDICATE BELOW THE EXTENT AND FREQUENCY OF YOUR NEWS RELATED ACTIVITIES

NEWS IS DEFINED AS INFORMATION ON EVENTS AND ISSUES THAT INCLUDE MORE THAN JUST FRIENDS AND FAMILY

SOCIAL MEDIA REFERS TO FACEBOOK, TWITTER OR SIMILAR

TRADITIONAL NEWS MEDIA REFERS TO TELEVISON, NEWS WEBSITE, NEWSPAPER AND RADIO 


\section{[RELATIVE ADVANTAGE OF SOCIAL BROADCASTING NETWORKS]}

Question: I use social media for news because...

\begin{tabular}{|l|l|l|l|l|}
\hline \multicolumn{1}{|c|}{ ITEMS } & \multicolumn{3}{|c|}{ SCALE } \\
\hline $\begin{array}{l}\text { RA1: The news is more current (e.g. breaking news, } \\
\text { headline news) compared to traditional news mediums } \\
\text { (e.g. T.V) }\end{array}$
\end{tabular}

\section{[USER INFLUENCE IN SOCIAL BROADCASTING NETWORKS]}

Question: I get my news through social media...

\begin{tabular}{|c|c|c|c|c|c|c|c|}
\hline \multirow[t]{3}{*}{ ITEMS } & \multicolumn{7}{|c|}{ SCALE } \\
\hline & \multicolumn{2}{|c|}{ NEVER } & \multicolumn{3}{|c|}{ SOMETIMES } & \multicolumn{2}{|c|}{ ALWAYS } \\
\hline & 1 & 2 & 3 & 4 & 5 & 6 & 7 \\
\hline $\begin{array}{l}\text { Ul1: By reading, browsing or surfing the news tweeted } \\
\text { or posted by others }\end{array}$ & $\boldsymbol{\square}$ & - & - & - & घ & $\boldsymbol{\square}$ & - \\
\hline $\begin{array}{l}\text { U12: By following tweets or post of reporters from } \\
\text { news companies }\end{array}$ & $\square$ & $\boldsymbol{\square}$ & $\square$ & & $\square$ & घ & - \\
\hline $\begin{array}{l}\text { UI3: By following tweets or posts of people with expert } \\
\text { insights, knowledge or reputation (e.g. celebrities, } \\
\text { politicians, CEOs) }\end{array}$ & $\boldsymbol{\square}$ & & $\boldsymbol{\square}$ & - & - & $\square$ & 口 \\
\hline $\begin{array}{l}\text { UI4: By reading, browsing or surfing the news tweeted } \\
\text { or posted only by news companies }\end{array}$ & 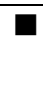 & - & $\square$ & 口 & $\mathbf{\square}$ & 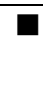 & 口 \\
\hline
\end{tabular}




\section{[CONTINUING NEWS CONSUMPTION IN SOCIAL BROADCASTING NETWORKS]}

Question: When I see news from news companies on social media or when my friends post or tweet news on social media...

\begin{tabular}{|c|c|c|c|c|c|c|c|}
\hline \multirow[t]{3}{*}{ ITEMS } & \multicolumn{7}{|c|}{ SCALE } \\
\hline & \multicolumn{2}{|c|}{ NEVER } & \multicolumn{3}{|c|}{ SOMETIMES } & \multicolumn{2}{|c|}{ ALWAYS } \\
\hline & 1 & 2 & 3 & 4 & 5 & 6 & 7 \\
\hline $\begin{array}{l}\text { CNT1: I check my friend's tweets or posts for more } \\
\text { information or for verifying the news }\end{array}$ & 口 & $\mathbf{\square}$ & $\mathbf{\square}$ & 口 & 口 & $\square$ & - \\
\hline $\begin{array}{l}\text { CNT2: I read the news and then share it (e.g. re-tweet } \\
\text { or re-post) on social media }\end{array}$ & - & $\boldsymbol{\square}$ & $\mathbf{\square}$ & $\square$ & 口 & $\square$ & $\boldsymbol{\square}$ \\
\hline $\begin{array}{l}\text { CNT3: I follow tweets or posts of people with expert } \\
\text { insights, knowledge or reputation (e.g. celebrities, } \\
\text { politicians, CEOs) }\end{array}$ & a & 口 & 口 & 口 & - & $\boldsymbol{\square}$ & घ \\
\hline $\begin{array}{l}\text { CNT4: I comment on the news that my friends post or } \\
\text { tweet }\end{array}$ & a & a & 口 & 口 & $\square$ & 口 & $\square$ \\
\hline $\begin{array}{l}\text { CNT5: I click 'like' on the news that my friends post or } \\
\text { tweet }\end{array}$ & a & a & a & $\mathbf{\square}$ & a & a & $\square$ \\
\hline
\end{tabular}

\section{[SWITCHING NEWS CONSUMPTION TO TRADITIONAL NEWS MEDIUMS]}

Question: When I see news from news companies on social media or when my friends post or tweet news on social media...

\begin{tabular}{|c|c|c|c|c|c|c|c|}
\hline \multirow[t]{3}{*}{ ITEMS } & \multicolumn{7}{|c|}{ SCALE } \\
\hline & \multicolumn{2}{|c|}{ NEVER } & \multicolumn{3}{|c|}{ SOMETIMES } & \multicolumn{2}{|c|}{ ALWAYS } \\
\hline & 1 & 2 & 3 & 4 & 5 & 6 & 7 \\
\hline $\begin{array}{l}\text { SWT1: I go to their traditional news mediums (e.g. TV } \\
\text { channels) for more information }\end{array}$ & - & - & $\mathbf{\square}$ & $\boldsymbol{\square}$ & $\boldsymbol{\square}$ & $\mathbf{\square}$ & $\boldsymbol{\square}$ \\
\hline $\begin{array}{l}\text { SWT2: I watch TV, read newspapers, listen to radio, } \\
\text { surf or browse news websites to verify the news }\end{array}$ & $\boldsymbol{\square}$ & $\mathbf{\square}$ & $\mathbf{\square}$ & 口 & 口 & $\square$ & $\boldsymbol{\square}$ \\
\hline $\begin{array}{l}\text { SWT3: I search traditional news mediums (e.g. T.V, } \\
\text { news websites) for more information }\end{array}$ & $\boldsymbol{\square}$ & $\boldsymbol{\square}$ & $\boldsymbol{\square}$ & घ & $\boldsymbol{\square}$ & $\boldsymbol{\square}$ & $\square$ \\
\hline $\begin{array}{l}\text { SWT4: I watch, listen, read, browse news on traditional } \\
\text { news mediums (e.g. TV or news websites) for more } \\
\text { information }\end{array}$ & & 口 & $\mathbf{\square}$ & 口 & 口 & $\mathbf{D}$ & $\boldsymbol{\square}$ \\
\hline
\end{tabular}




\section{[COMPARATIVE DURATION OF NEWS CONSUMPTION ON SOCIAL} BROADCASTING NETWORKS]

Question: Please indicate the comparative time you spend on social media

\begin{tabular}{|c|c|c|c|c|c|c|c|}
\hline \multirow[t]{3}{*}{ ITEMS } & \multicolumn{7}{|c|}{ SCALE } \\
\hline & \multicolumn{2}{|c|}{ NEVER } & \multicolumn{3}{|c|}{ SOMETIMES } & \multicolumn{2}{|c|}{ ALWAYS } \\
\hline & 1 & 2 & 3 & 4 & 5 & 6 & 7 \\
\hline $\begin{array}{l}\text { CNC1: I spend more time every day reading news on } \\
\text { social media compared to traditional news mediums } \\
\text { (e.g. T.V or news websites) }\end{array}$ & घ & 口 & $\boldsymbol{\square}$ & - & घ & $\boldsymbol{\square}$ & $\mathbf{\square}$ \\
\hline $\begin{array}{l}\text { CNC2: I spend more time every day checking news on } \\
\text { social media compared to traditional news mediums } \\
\text { (e.g. T.V or news websites) }\end{array}$ & घ & 口 & $\boldsymbol{\square}$ & - & घ & $\boldsymbol{\square}$ & घ \\
\hline $\begin{array}{l}\text { CNC3: I spend more days in a week for all my news } \\
\text { related activities (e.g. reading, commenting, tweeting } \\
\text { or posting) on social media compared to reading, } \\
\text { watching or listening to news on traditional news } \\
\text { mediums (e.g. T.V or news websites) }\end{array}$ & घ & च & - & $\boldsymbol{\square}$ & च & $\boldsymbol{\square}$ & $\mathbf{\square}$ \\
\hline
\end{tabular}

Copyright: $@ 2018$ Palekar \&Sedera. This is an open-access article distributed under the terms of the Creative Commons Attribution-NonCommercial 3.0 Australia License, which permits non-commercial use, distribution, and reproduction in any medium, provided the original author and AJIS are credited.

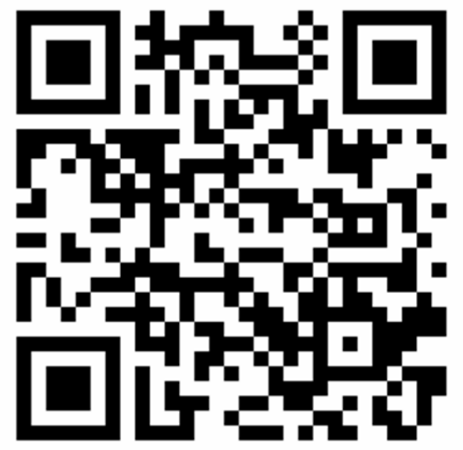

